\title{
Deep Trek: Mission Concepts for Exploring Subsurface Habitability \& Life on Mars A Window into Subsurface Life in the Solar System
}

\section{Lead Author:}

Charles D. Edwards (Jet Propulsion Laboratory, California Institute of Technology).

Contact: Charles.D.Edwards@jpl.nasa.gov

\section{Co-Authors:}

1. Vlada Stamenković

2. Penelope Boston

3. Kennda Lynch

4. Jesse Tarnas

5. Barbara Sherwood-Lollar

6. Sushil Atreya

7. Alexis Templeton

8. Anthony Freeman

9. Woodward Fischer

10. Tilman Spohn

11. Chris Webster

12. Alberto G. Fairén

13. John (Jack) Mustard

14. Michael Mischna

15. Tullis C. Onstott

16. Magdalena Rose Osburn

17. Thomas Kieft

18. Robert E. Grimm

19. William B. Brinckerhoff

20. Sarah Johnson

21. Luther Beegle

22. James Head

23. Albert Haldemann

24. Charles Cockell

25. Jobn Hernlund

26. Brian Wilcox

27. David Paige

28. Giuseppe Etiope

29. Daniel Glavin

30. Maria-Paz Zorzano

31. Yasubito Sekine

32. Stalport Fabien

33. Joseph Kirschvink

34. Cara Magnabosco

35. Roberto Orosei
Jet Propulsion Laboratory, California Institute of Technology

NASA Ames

LPI/USRA

Brown University

University of Toronto

University of Michigan

University of Colorado

Jet Propulsion Laboratory, California Institute of Technology California Institute of Technology

International Space Science Institute

Jet Propulsion Laboratory, California Institute of Technology

Centro de Astrobiología (CSIC-INTA)

Brown University

Jet Propulsion Laboratory, California Institute of Technology

Princeton University

Northwestern University

NMT

$\int w \mathrm{R} I$

NASA Goddard

Georgetown University

Jet Propulsion Laboratory, California Institute of Technology

Brown University

ESA/ESTEC

University of Edinburgh

ELSI, Tokyo Tech

JPL/Marine Biomass

UCLA

Istituto Nazionale di Geofisica e Vulcanologia, Roma, Italy

NASA Goddard Space Flight Center

Centro de Astrobiología (CSIC-INTA)

ELSI, Tokyo Tech

LISA

California Institute of Technology

ETH

Istituto Nazionale di Astrofisica 


\section{Deep Trek: Missions Concepts for Exploring Subsurface Habitability \& Life on Mars}

36. Matthias Grott

37. John D. Rummel

38. Atsuko Kobayashi

39. Fumio Inagaki

40. Janice Bishop

41. Vincent Cheurier

42. Mary Sue Bell

43. Beth N. Orcutt

44. Jennifer McIntosh

45. Katarina Miljkovic

46. Doris Breuer

47. Tomobiro Usui

48. Kris Zacny

49. Essam Heggy

50. Edgard G. Rivera-Valentín

51. Nathan J. Barba

52. Ryan Woolley

53. Oliver Warr

54. Mike Malaska

55. Jennifer G. Blank

56. Donald F. Ruffatto

57. Haley M. Sapers

58. Larry H. Matthies

59. Lewis Ward

60. Svetlana Shkolyar

61. Cedric Schmelzbach

62. Travis S.J. Gabriel

63. Ceth Parker

64. Hermes Hernan Bolivar-Torres

65. Bernadett Pál

66. Dirk Schulze-Makuch

67. Jorge Andres Torres Celis

68. Akos Kereszturi

69. J. Andy Spry

70. Kyle Uckert

71. Marc A. Hesse

72. Rachel Harris

73. Ana-Catalina Plesa

74. Rепуи Hu

75. Ali-akbar Agha-mohammadi

76. Brian D. Wade

77. Snehamoy Chatterjee

78. Patrick McGarey

79. Heather Valeah Graham

80. Shino Suzuki

81. Matt Schrenk

82. Kristopher Sherrill

83. Scott Howe
DLR

Friday Harbor Partners LLC

Earth-Life Science Institute, Tokyo institute of Technology

JAMSTEC

SETI Institute

University of Arkansas

Jacobs@NASA/Johnson Space Center

Bigelow Laboratory for Ocean Sciences

University of Arizona

Curtin University

DLR

$J A X A$

Honeybee Robotics

University of Southern California

Lunar and Planetary Institute (USRA)

Jet Propulsion Laboratory, California Institute of Technology

Jet Propulsion Laboratory, California Institute of Technology

University of Toronto

Jet Propulsion Laboratory, California Institute of Technology

NASA Ames/Blue Marble Space Institute of Science

Jet Propulsion Laboratory, California Institute of Technology Caltech/USC/JPL

Jet Propulsion Laboratory, California Institute of Technology

Harvard University

NASA GSFC/USRA

ETH Zurich, Switzerland

Arizona State University

Jet Propulsion Laboratory, California Institute of Technology

Universidad Nacional Autónoma de México

CSFK KTM CSI

Technical University Berlin

Universidad Nacional Autónoma de México

Research Centre for Astronomy and Earth Sciences

SETI Institute

Jet Propulsion Laboratory, California Institute of Technology

The University of Texas at Austin

Harvard University

DLR

Jet Propulsion Laboratory, California Institute of Technology

Jet Propulsion Laboratory, California Institute of Technology

Michigan State University

Michigan Technological University

Jet Propulsion Laboratory, California Institute of Technology

NASA GSFC

JAMSTEC

Michigan State University

Jet Propulsion Laboratory, California Institute of Technology

Jet Propulsion Laboratory, California Institute of Technology 


\section{Deep Trek: Missions Concepts for Exploring Subsurface Habitability \& Life on Mars}

84. Raju Manthena

85. Mariko Burgin

86. Kalind Carpenter

87. Louis Giersch

88. Velibor Cormarkovic

89. Nigel Smith

90. Jeffrey J. McDonnell

91. Joseph Michalski

92. Devanshu Jha

93. Morgan L. Cable

94. Elodie Gloesener

95. Varun Paul

96. Stewart Gault

97. Sharon Kedar

98. Eloise Martean

99. Orkun Temel

100.Seth Krieger

101.Ryan Timoney
Jet Propulsion Laboratory, California Institute of Technology Jet Propulsion Laboratory, California Institute of Technology Jet Propulsion Laboratory, California Institute of Technology Jet Propulsion Laboratory, California Institute of Technology Jet Propulsion Laboratory, California Institute of Technology Snolab

University of Saskatchewan

University of Hong Kong

MVJ College of Engineering

Jet Propulsion Laboratory, California Institute of Technology

UCLouvain

Mississippi State University

University of Edinburgh

Jet Propulsion Laboratory, California Institute of Technology Jet Propulsion Laboratory, California Institute of Technology

Royal Observatory of Belgium

USC

University of Glasgow

LINK to List of Co-Authors \& Co-Signatories:

bttps:// drive.google.com/file/d/1qogP3k.AubSt918v1MSoBvqtTIYNQE_cS/view?usp=sharing 


\section{Motivation: An Unprecedented Scientific Opportunity}

A companion white paper entitled "Deep Trek: Science of Subsurface Habitability \& Life on Mars" highlights the immense science return potential of Mars subsurface exploration. Here we focus on mission concepts, driving science objectives, and technologies that enable access to a major new frontier in planetary science, the Martian subsurface, with a focus on modern subsurface habitability \& life. Different mission architectures spanning small spacecraft (SSC), Discovery, New Frontiers, to Flagship classes will generate major discoveries relating to the potential for life in the Martian subsurface.

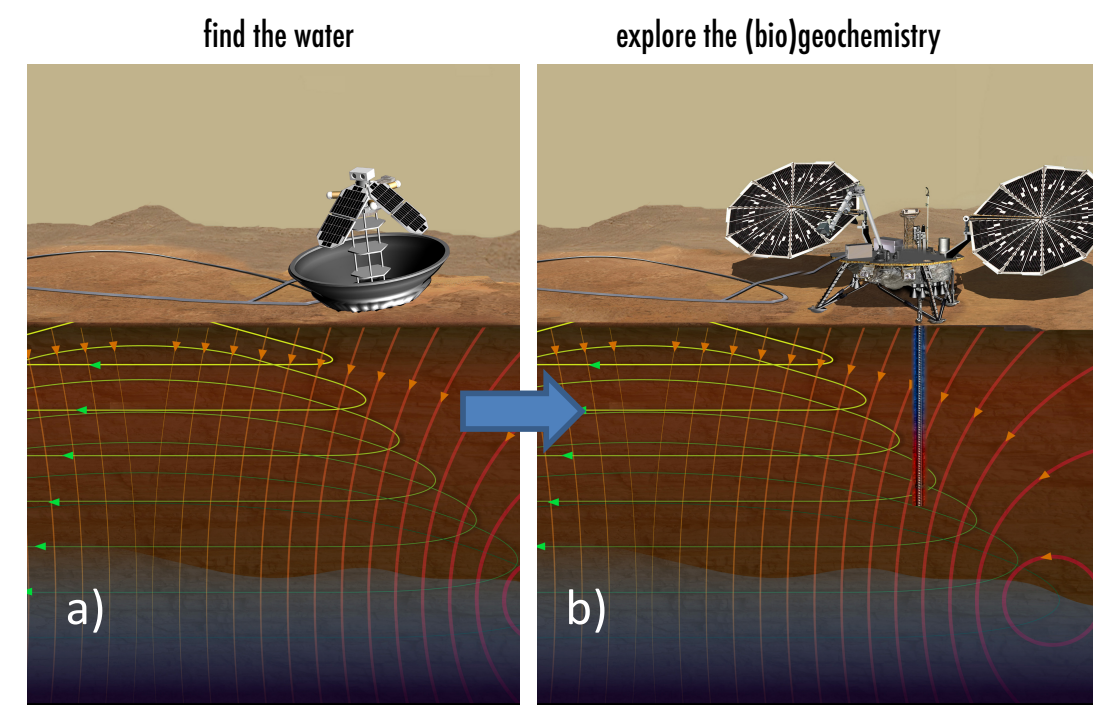

Fig. 1: Exploration of the Martian subsurface with SSCs, New Frontiers \& Flagship mission concepts using the $V A L K Y R I E$ concepts. SSCs with liquid water sounders can detect groundwater to depths of many kilometers (a). New Frontiers-type mission concepts would allow more advanced \& complete characterization of subsurface habitability with drills accessing samples to depths of 10-100 $m(b)$.

The architectures shown here belong to a class of mission concepts called VALKYRIE: "Volatiles $\underline{\text { And }} \underline{\mathbf{L}}$ ife: $\underline{\mathbf{K}} \underline{\mathbf{Y}} \underline{\mathbf{R}}$ econnaissance \& $\underline{\mathbf{I}} n$-Situ Exploration". Building a bridge over four decades from the first daring mission that searched for signs of extant life on Mars with the 1976 Viking landers, the VALKYRIE mission concepts re-address the question of whether there could be habitable environments and/or life on Mars today. However, VALKYRIE focuses on the most likely potential modern habitable environment, the Martian subsurface, implementing cutting-edge technologies \& leveraging our scientific understanding of Mars and terrestrial subsurface life, all of which have greatly improved since the Viking mission era.

\section{Science Goals \& Objectives}

The mission concepts proposed here aim to respond to some or to all of the two science goals specified in our companion paper. These science goals are: (G1) quantify modern Martian subsurface habitability and (G2) search for evidence of extant subsurface life. Our Mission Goal (1), subsurface habitability exploration, has three objectives (see our Science Traceability Matrix, STM in Fig. 3): (A) locate \& characterize liquid water, (B) identify energy \& nutrient sources, and (C) assess molecular and cellular stability potential with depth. Whether liquid water exists on Mars today is explored with the first objective. The second objective focuses on the availability of redox nutrients and energy gradients that could drive microbial metabolic activity by determining the (bio)geochemical and mineralogical state of the subsurface. The third objective investigates molecular and cellular stability, by determining how deep ionizing radiation and oxidizing radicals penetrate into the Martian subsurface. This leads to a fourth objective, directly addressing Mission Goal 2, to (D) search for signs of extant subsurface life by looking for biomarkers \& signs of metabolic activity. Landing sites have been discussed in our companion paper "Deep Trek: Science of Subsurface Habitability \& Life on Mars". Our mission concepts are also linked to secondary science goals such as seeking signs of extinct life, reconstructing climate history, geophysical structure of the crust, resource prospecting \& human exploration (see Stamenković et al., 2019). 
3. Science Traceability \& Objectives

3.1. Objective A: Is There Groundwater Today?

Liquid water with sufficiently high water activity is essential for life as we know it. On Mars, following the (liquid) water leads us to depths of many kilometers. The proposed investigations would determine the amount of adsorbed subsurface water as a function of depth, as well as the depth, thickness, and chemistry of the putative groundwater table hypothesized to exist many kilometers below the surface (A1, A2). This may be achieved by using a transient electromagnetic sounder (TEM) from the surface without any drilling, which measures the electrical conductivity of the subsurface and inverts those data into profiles of liquid water abundance, hydration and salinity and has been one of the most common techniques to search for groundwater on the Earth in the last three decades. The electrical conductivity will help us to determine the concentration of ions in the groundwater by relating the measured electrical conductivity to potential ionic compositions. Measurements of the subsurface geothermal gradient with depth (A5 $\rightarrow$ M3, M7) will help further constrain the composition of the groundwater through comparison with data for freezing-point depression in the presence of salts. Measuring the porosity change with depth will allow us to extrapolate, beyond the accessible drilling depth, the water storage capacity and the physically habitable space available (A3). It is important to note that even in the unlikely case of a local non-detection of groundwater, this finding would allow us to put constraints on global water inventories (in combination with measurements of the porosity, the unaltered hydration state and the salt content with depth) and be a significant and surprising discovery by itself.

\subsection{Objective B: Geochemical Gradients with Depth}

The proposed investigations would allow us to determine the geochemical (B1) and mineralogical (B2) properties of the Martian subsurface to depths of at least $10 \mathrm{~m}$ (threshold) but aiming for $100 \mathrm{~m}$ (baseline). Drilling to $10 \mathrm{~m}$ would be sufficient to mitigate surficial temperature variations (below $\sim 5$ $\mathrm{m})$, to test hypotheses on cellular degradation as a function of depth due to presence of chaotropic oxidants (e.g., perchlorates) and ionizing radiation, and to be conservatively far enough from the highly oxidizing and radiation-intense surface environment to observe physico-chemical conditions more representative of an isolated deeper subsurface. However, drilling to $\sim 100 \mathrm{~m}$ would increase scientific return considerably by permitting measurements of larger-scale redox gradients, which would enable development of new water inventory and subsurface habitability models. This will form the basis for extrapolating measurements and models down to regions at kmdepths where groundwater with a higher water activity is more likely (which VALKYRIE would not access physically). Of particular focus will be the characterization of chemical redox gradients and disequilibria, and specifically the oxidation states of S, Fe, N and Mn compounds in soil (M8) and minerals (M9) as a function of depth, unaltered from hostile surface conditions. The Fe-Mn pair provides especially good constraints on redox conditions and water availability with depth, as Fe and $\mathrm{Mn}$ need very different concentrations of oxidants and liquid water to be oxidized. Exposed scarps on the surface and shallow caves are continuously altered by the atmosphere and do, hence, not provide the insight that deep vertical drilling provides.

\subsection{Objective C: Cellular Stability with Depth}

Proposed investigations would test existing hypotheses and models of the abundance of oxidants (C1) and ionizing radiation (C2) as a function of depth. By accessing samples to at least $\sim 10 \mathrm{~m}$, we include the worst-case predictions of the depth where cells could still sustain damage (Dartnell et al., 2007).

\subsection{Objective D: Whiffs \& Fingerprints of Extant Life}

We propose a threefold strategy for searching for signs of extant life in the Martian subsurface. This focuses on organic (D1) and inorganic (D2) indicators of life, by measuring the relative abundances 
of amino acids, lipids, biomolecules, metabolic byproducts, isotopic signatures in multiple organic and inorganic carbon compounds, and biominerals and microstructures. The change in depth of these biosignatures, especially in relation to variations in mineral hydration states, will help us explore whether we see indications of a deep biosphere. Adding sampling of trace gases or volatile metabolic byproducts (D3) and their variability with depth and time will provide further information regarding the likelihood of observing the first evidence of extant subsurface life.

\section{Instruments}

\subsection{Groundwater Sounders: Find the Liquid Subsurface Water Without Drilling}

The most plausible method at present for groundwater detection to depths of many kilometers is transient electromagnetic (TEM) sounding from a planet's surface (orbiting assets cannot address the existence of groundwater on Mars at the sounding depths that are needed), which operates at lower frequencies than radar measurements, thereby penetrating far deeper. The Transmissive $\underline{\mathrm{H}}_{2} \mathrm{O}$ Reconnaissance $\left(\mathrm{TH}_{2} \mathrm{OR}\right)$ TEM instrument under development at JPL has achieved a TRL of 4 with anticipated TRL of 6 by 2023, <10 kg, 3u, <500 Wh/sol). This low-mass instrument is key for a subsurface mission to Mars of any budget class, as it will robustly test for the presence of groundwater and determine the physical and chemical properties of groundwater reservoirs that extant Martian life could inhabit. Penetration depth into the Martian subsurface by the orbiting radar MARSIS on Mars Express and SHARAD on MRO has likely been limited to depths of $\sim 100-200 \mathrm{~m}$, except for "regions with favorable subsurface conditions" with ice or volcanic ash (see Stillman \& Grimm, 2011). Ground penetrating surface radar and a seismic station can assist TEM sounders to better constrain groundwater inventories, by determining distinct subsurface layers.

\subsection{Drills: Access Subsurface (Bio)Geochemical Gradients}

The drill on Curiosity has been used to access depths of $\sim 7 \mathrm{~cm}$ and the Perseverance drill is designed to reach similar depths. Drills reaching 1-10 $\mathrm{m}$ in typical Mars subsurface materials (competent \& friable rocks) have been demonstrated under simulated or Mars analogue conditions at TRLs of 5-6 and are compatible with New Frontiers-class lander missions (e.g., ExoMars Drill, Icebreaker, MARTE, Planetary Deep Drill [PDD]). The PDD wireline drill (at TRL 5 and $70 \mathrm{~kg}$ ) from Honeybee Robotics has drilled $13.5 \mathrm{~m}$ into gypsum (drilling could have progressed deeper but was balted due to limited funding).

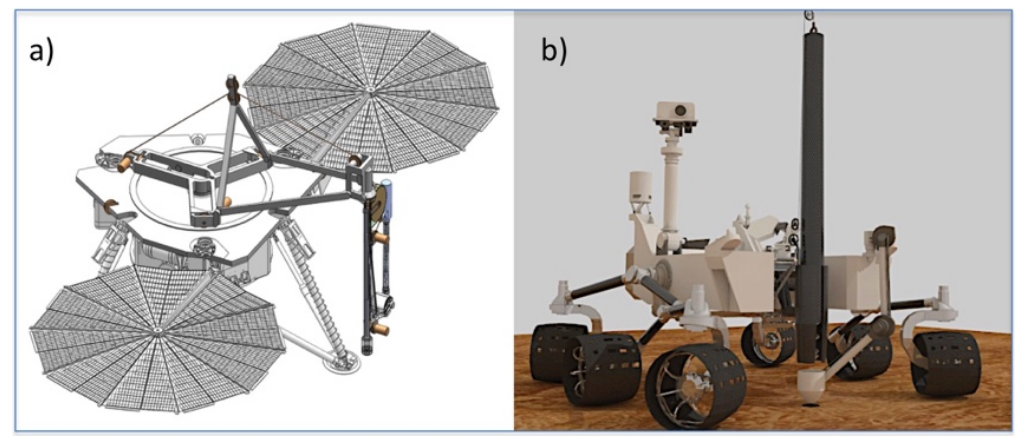

Fig. 2: (a) Wireline drill ASGARD. Drilling is feasible to $100 \mathrm{~m}$ with New Frontiers cap on InSight-like platform. (b) Mobile PDD.

To get to depths of $\sim 100 \mathrm{~m}$ and more, while still remaining compatible with a New Frontiers class lander mission, wireline drilling approaches are appealing: PDD, for example, was tested successfully in 2019 to a depth of $111 \mathrm{~m}$ in ice (Eshelmann et al., 2019; Bhartia et al., 2019), which has a similar

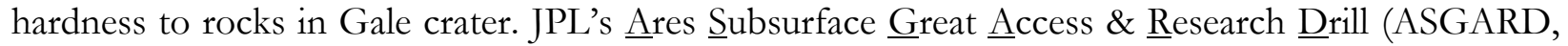
Fig. 2a, TRL 5 targeted in 2020) is another miniaturized wireline drill with a target mass below $50 \mathrm{~kg}$, using on average less than $100 \mathrm{~W}$ of solar power and compressed $\mathrm{CO}_{2}$ harvested in situ from the Martian atmosphere as drilling fluid. For both wireline drills, cuttings would be delivered in stratigraphic order with a resolution capability of $\sim$ centimeters. Using in-borehole instruments (as for an enbanced mission scenario) can increase this stratigraphic resolution to $\mathrm{mm}$ (this has been demonstrated by PDD in Greenland). The advantage of wireline drills is that drilling can proceed to depths much greater than $\sim 100 \mathrm{~m}$ without any significant increase in instrument mass- 
providing the opportunity to go beyond $100 \mathrm{~m}$ in an extended mission mode (the only cost to go deeper is the mass of the tether, which is $<1 \mathrm{~kg} / \mathrm{km}$ of tether, and the time for drilling). One challenge of wireline systems is that drilling must occur in geologically competent material to prevent borehole collapse. Fortunately, (i) such materials (including volcanic and sedimentary lithologies) are common and widespread on Mars, (ii) they are well-suited targets for astrobiological subsurface science, and (iii) borehole stability is generally not an issue in such rocks. For example, the $13.5 \mathrm{~m}$ borehole drilled by PDD in a gypsum quarry in 2015 is still open. Boreholes in petroleum/natural gas injection and production wells, and those from exploration drilling in the mining sector, often remain open for decades. On Mars, due to the lower gravity and the lower seismic stresses, boreholes should remain stable to depths of hundreds of meters (Zacny \& BarCohen, 2009). Lastly, all the 100+ $\mathrm{m}$ drills discussed here, including coiled tubing drills like RedWater that can drill in any kind of soil, are compatible with Curiosity-class rovers that would be Flagship class missions (Fig. 2b for PDD). Note: InSight's HP' mole (Spohn et al., 2018) was designed for penetration through loose soil. A mole is not baselined for any of the missions proposed here.

\subsection{Bio-geochemical/Radiation Analysis Suite}

A multi-analytical approach to assessing the spatially correlated mineralogical, chemical, and molecular complexity of a prospective habitable environment remains the most promising strategy for biosignature detection. Mass spectrometry, often coupled with gas chromatography, is a proven technique for detection of volatile and refractory organics in planetary environments. Mass spectrometry can be coupled with various front-end sample introduction techniques including lasers for spatially resolved compositional measurements and capillary electrophoresis for water soluble organics. The most recent examples of mass spectrometers designed for flight are the Sample Analysis at Mars (SAM) instrument (Mahaffy et al., 2012) on Curiosity and the Mars Organic Molecule Analyzer Mass Spectrometer (MOMA-MS, Brinckerhoff et al., 2013), which is flight qualified (TRL 8). Mass spectrometry also detects the lower molecular weight volatiles representing products of respiration of extant life such as $\mathrm{CH}_{4}, \mathrm{CO}_{2}, \mathrm{~N}_{2}, \mathrm{H}_{2}, \mathrm{O}_{2}$, and non-methane hydrocarbons (Fig. 3, B1, M8; C1, M8). The SAM quadrupole mass spectrometer has demonstrated stable isotopic measurements of carbon (Mahaffy et al., 2013), nitrogen (Wong et al., 2013), and sulfur (Franz et al., 2017), thus meeting the stable isotopes measurement requirement (Fig. 3, D1, M12). IR spectroscopy, such as the Tunable Laser Spectroscopy (TLS) on the SAM instrument, is a high TRL, proven technique for measuring the abundance and isotopic composition of trace gases of biological interest (e.g., Webster et al., 2018) that complements mass spectrometry and other compositional analyzers. Additional instruments may include the Wet Chemistry Laboratory (WCL) deployed on Phoenix for redox chemistry identification, chemical mapping through X-ray and fluorescence spectrometry such as PIXL, chemical analysis through laser-induced breakdown spectroscopy (LIBS) (ChemCam, SuperCam), organic mapping with Raman spectrometers (SHERLOC) and mineralogy with imaging spectrometers. Deep UV Raman spectroscopy is especially intriguing due to its ability to detect small concentrations of aromatic organics. An on-board radiometer/ high-energy particle tracker would allow for characterization of ionizing radiation levels with depth.

\section{Mission Concept Architectures}

Because the (bio)geochemistry of the subsurface of Mars has never been explored in detail, the aforementioned instruments can be mixed-and-matched depending on the level of funding available for a given mission. All budget classes have the capacity for significant discovery and advancement of knowledge regarding subsurface habitable environments on Mars and the possibility of extant subsurface life. The information below on mission cost is an outcome of an architecture Team $X$ study at JPL and work by our internal JPL SSC Team performed in 2018-2020 (Barba et al., 2020). 


\subsection{SSCs: Find the Groundwater}

We can assess the presence of groundwater from the Martian surface at low cost in this decade. As described in the Decadal Whitepaper by Barba et al. (2020), small spacecraft are an especially important opportunity for Mars subsurface science. We have found that, using fully hosted piggyback opportunities, such as with Mars Sample Return or Ice Mapper planned for 2026, we can answer some of the leading objectives (groundwater detection \& characterization [A1-A2] as well as trace gas localization [D3]) at an A-D cost of $\sim \$ 120 \mathrm{M}$ total. To achieve such goals, as a priority, this mission would deliver a TEM liquid water sounder to the Martian surface with a rough lander such as SHIELD (see Barba et al. 2020) or the similar TRL 6 Finnish Lander. If launched as a secondary, or primary payload on a dedicated launch vehicle such as Falcon 9, independently making its way to Mars, then A-D costs for one small lander mission would be $\sim \$ 150-200 \mathrm{M}$ without launch (plus up to $\sim \$ 60 \mathrm{M}$ for launch costs, e.g., with Falcon 9). If three SHIELD landers, carrying the payload mentioned above, would be merged on a Falcon 9 under Class D requirements, then the costs for A-D would mount to $\sim \$ 300 \mathrm{M}+$ launch costs. This multi-unit option improves our ability to extrapolate local results to global water inventories and facilitates a price/unit drop by $\sim \$ 50-100 \mathrm{M}-$ which could open up doors for sharing with international and commercial partners.

\subsection{Discovery Class: Multiple SSCs Rough Landers or One InSight-type Lander}

The latter 3-unit rough lander concept, for example, carrying a groundwater sounder like $\mathrm{TH}_{2} \mathrm{OR}$, would fit as well under the Discovery cap even if system assurance is increased to a Class B mission. One single heritage-based InSight-type lander could also deliver one groundwater sounder within the Discovery budget. The InSight-type concept leverages a high heritage and low risk landing system, while the SSC rough lander concept has the advantage of delivering the same instrument at three different locations for a similar price as one single InSight lander, probing the unexplored subsurface in different geologic contexts and enhancing the science return by providing a more global perspective on groundwater distribution.

\subsection{New Frontiers Class: Physical Access to the Subsurface is Feasible}

TEM sounding from the surface for groundwater is a critical objective for the VALKYRIE mission concepts but the science return is significantly enhanced once a drill is added to sample subsurface redox gradients, going beyond objectives A1 and A2. We consider a basic instrument suite such as one TEM, two ICC surface context cameras, a MOMA GC-MS, a TLS, a MET Station, a High Energy Particle Tracker and a heat probe connected to the drill. To minimize planetary protection protocols and total mass, we assume that all baseline instruments will be on the surface as part of an analysis suite, with samples being delivered as they are being drilled. We find that by using an InSight-type platform, we can address all the science goals of VALKYRIE, within a New Frontiers-type budget. However, it is crucial that the drill remains below $50 \mathrm{~kg}$. This is feasible for a $10 \mathrm{~m}$ Honeybee coil tubing drill like RedWater, which could operate in any kind of ground. This mass limit is also in reach for a $100 \mathrm{~m}$ wireline drill like JPL's ASGARD with near-term investments (Fig. 2a).

\subsection{Flagships: Vertical Exploration with Mobility}

Mobility offers the advantage of multiple drill sites but moves the cost into the Flagship Class domain. The class of drills proposed here $(10 \mathrm{~m}$ and $100 \mathrm{~m})$ is compatible with Curiosity/Perseverancetype rovers (see Fig. 2b for a $100 \mathrm{~m}$ PDD drill system). The complete enhanced VALKYRIE sample analysis payload, as described in Fig. 3, is in many ways similar to the payload on Curiosity/Perseverance. In comparison to our New Frontiers-type baseline scenario, our Flagship baseline payload includes additionally a Deep UV Raman instrument (SHERLOC) and the ICC camera is replaced with ChemCam/SuperCam. In an enhanced mission scenario, an additional TLS can be added as a down- 


\section{Deep Trek: Missions Concepts for Exploring Subsurface Habitability \& Life on Mars}

borehole device to provide better constraints on subsurface trace gases gradients. Additional instruments as shown in Fig. 3 can be included for an enhanced mission scenario.

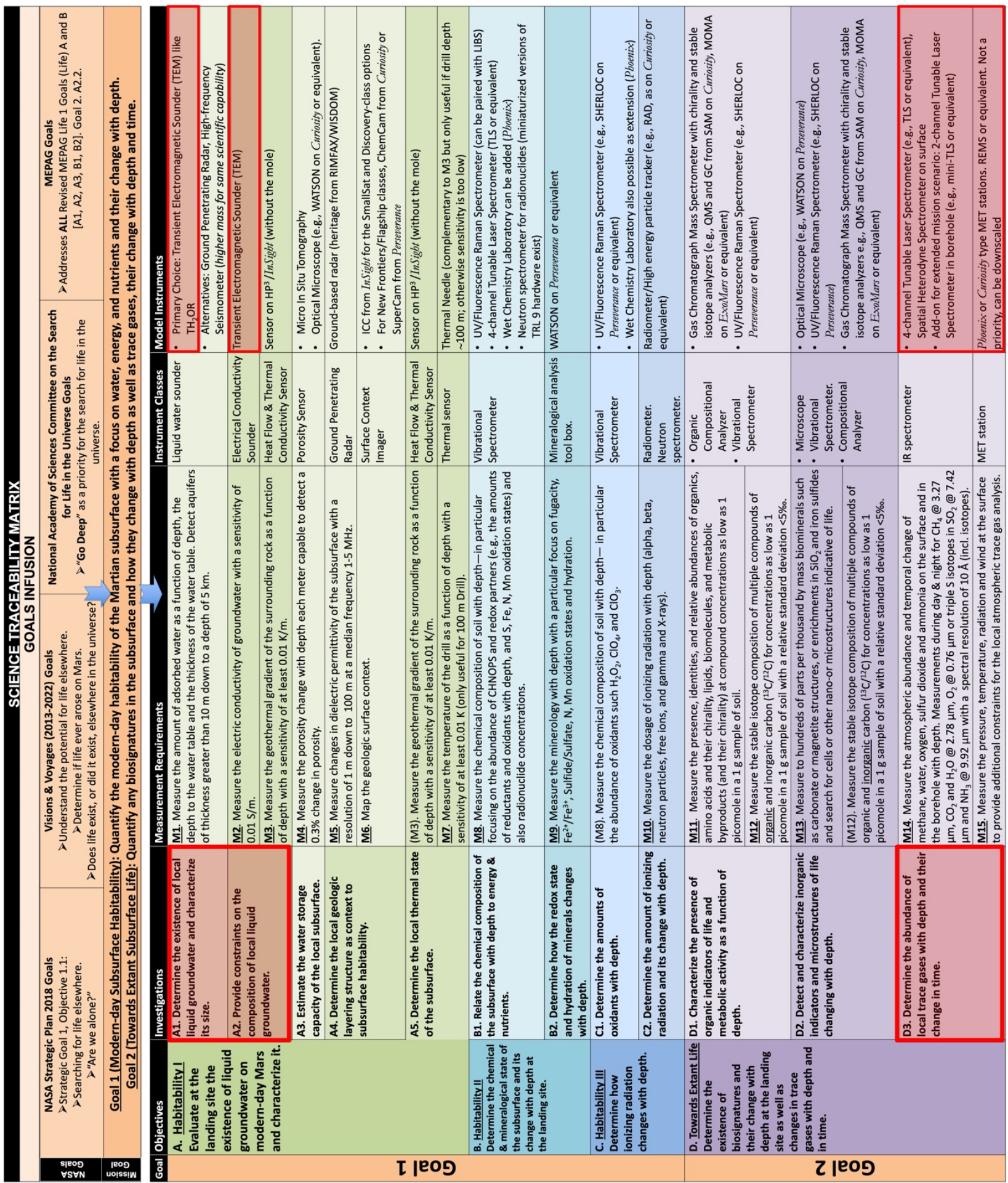

Fig. 3: Science Traceability Matrix (STM). Identical measurements are needed for a few objectives; if so, they are shown in brackets, e.g., (M3, M8 \& M12) for A5, C1 \& D2. The red boxes indicate investigations that can be addressed without drilling with SSCS (groundwater characterization with sounders and trace gas localization). All other investigations require drilling of 10-100 m (100 m being the baseline target). 


\section{Planetary Protection}

Planetary protection would follow the relevant NASA policies, keeping in mind the Viking mission guidelines but with additional requirements to remove left-over organics such as amino acids, nucleobases, and carboxylic acids to minimize the total exogenous organic carbon load. By limiting the use of in-borehole instruments (as in our baseline scenario), only the drill, the sample delivery system, and the $\mathrm{CO}_{2}$ pumping system (for wirelines) would need to be sterilized. This is feasible because the components are steel/titanium based with no electronics. Dry Heat Microbial Reduction or various types of gas sterilization can also be used to sterilize non steel/titanium components.

\section{Synergy with Commercial \& Human Exploration}

Access to water in the form of hydrated salts, ice or potentially groundwater is key for enabling human exploration and settlement of Mars. The same technologies employed by science-oriented Mars subsurface exploration missions will also enable characterization and utilization of these key waterbearing resources required by future human Mars explorers. Mars subsurface science therefore hosts unique potential for collaboration between SMD and HEOMD, as well as collaboration with international \& commercial partners who are interested in enabling human settlement of Mars.

\section{Conclusions}

Decades of Mars exploration by orbital and landed spacecraft, coupled with knowledge of Deep Life on the Earth, suggest that the subsurface of Mars may still host a long-lived habitable environment that could contain extant life. Exploring this potentially habitable subsurface environment, and searching for extant life there, is the natural progression of the last 2-3 decades that have been focused on ancient life, and is opening up a new exploration pathway that is complementary to MSR and the Perseverance rover's science goals. SSC options for characterizing groundwater and New Frontiers-type missions with drills to determine subsurface (bio)geochemistry are feasible. Mars is the natural testbed for subsurface exploration that will inevitably be applied to other, technologically more ambitious, targets such as the subsurfaces of icy moons or small bodies. The proposed mission concepts are not only relevant to NASA's overarching \& driving scientific goals, but tap into the primary reason the public finds planetary exploration exciting: the possibility of extant life on other worlds.

\section{References}

Barba et al., 2020. White Paper to The Decadal Survey in Planetary Science \& Astrobiology.

Bhartia et al., 2019. https://ui.adsabs.harvard.edu/abs/2019AGUFM.P53D3501B/abstract

Brinckerhoff, W. et al., 2013. IEEE. doi: 10.1109/AERO.2013.6496942.

Dartnell, L.R. et al., 2007. GRL 34. doi.org/10.1029/2006GL027494

Eshelman, et al., 2019. Astrobiology. 19. doi.org/10.1089/ast.2018.1925

Franz, H.B. et al., 2017. Nat. Geo. 10. doi.org/10.1038/ngeo3002

Mahaffy, P. et al., 2012, Space Sci. Rev. 170. doi.org/10.1007/s11214-012-9879-z

Mahaffym P. et al, 2013. Science 341. doi.org/10.1126/science.1237966

Spohn et al., 2018. Space Sci. Rev. 214. doi.org/10.1007/s11214-018-0531-4

Stamenković, et al., 2019. Nat. Ast. 3. doi.org/10.1038/s41550-018-0676-9

Stillman D. E. \& Grimm, R.E.. JGR 116. doi.org/10.1029/2010JE003661

Webster, C.R., et al., 2018. Sci. 360. doi.org/10.1126/science.aaq0131

Wong, M. et al., 2013. GRL 40. doi.org/10.1002/2013GL057840

Zacny, K. \& Bar-Cohen, Y., 2009. springer.com/gp/book/9783642036286

Part of this research was carried out at the Jet Propulsion Laboratory, California Institute of Technology, under a contract with the National Aeronautics and Space Administration (80NM0018D0004). Pre-Decisional Information - For Planning and Discussion Purposes Only. 